ARTICLE

\title{
Confining energy migration in upconversion nanoparticles towards deep ultraviolet lasing
}

Xian Chen ${ }^{1, \star}$, Limin $\mathrm{Jin}^{2, \star}$, Wei Kong${ }^{1}$, Tianying Sun ${ }^{1}$, Wenfei Zhang ${ }^{2}$, Xinhong Liu ${ }^{3}$, Jun Fan ${ }^{1,4}$, Siu Fung Yu ${ }^{2}$ \& Feng Wang ${ }^{1,4}$

Manipulating particle size is a powerful means of creating unprecedented optical properties in metals and semiconductors. Here we report an insulator system composed of $\mathrm{NaYbF}_{4}: \mathrm{Tm}$ in which size effect can be harnessed to enhance multiphoton upconversion. Our mechanistic investigations suggest that the phenomenon stems from spatial confinement of energy migration in nanosized structures. We show that confining energy migration constitutes a general and versatile strategy to manipulating multiphoton upconversion, demonstrating an efficient five-photon upconversion emission of $\mathrm{Tm}^{3+}$ in a stoichiometric $\mathrm{Yb}$ lattice without suffering from concentration quenching. The high emission intensity is unambiguously substantiated by realizing room-temperature lasing emission at around $311 \mathrm{~nm}$ after $980-\mathrm{nm}$ pumping, recording an optical gain two orders of magnitude larger than that of a conventional $\mathrm{Yb} / \mathrm{Tm}$-based system operating at $650 \mathrm{~nm}$. Our findings thus highlight the viability of realizing diode-pumped lasing in deep ultraviolet regime for various practical applications.

\footnotetext{
${ }^{1}$ Department of Physics and Materials Science, City University of Hong Kong, 83 Tat Chee Avenue, Hong Kong SAR, China. ${ }^{2}$ Department of Applied Physics, The Hong Kong Polytechnic University, Hung Hom, Hong Kong SAR, China. ${ }^{3}$ Department of Electronic Engineering, City University of Hong Kong, 83 Tat Chee Avenue, Hong Kong SAR, China. ${ }^{4}$ City University of Hong Kong Shenzhen Research Institute, Shenzhen 518057, China. * These authors contributed equally to this work. Correspondence and requests for materials should be addressed to S.F.Y. (email: sfyu21@hotmail.com) or to F.W. (email: fwang24@cityu.edu.hk).
} 
T he construction of functional materials with designable optical properties is fundamentally important for scientific research and technological applications in diverse fields encompassing energy, environment and biomedicine ${ }^{1-11}$. Given the constraints in designing materials using different combinations of elements, nanoscale manipulation of matters has become a promising alternative to the creation of novel functional materials ${ }^{12-19}$. Particularly, by taking the advantage of size confinement effects, the energy band structure in semiconductors can be precisely modified to offer size-tunable emission wavelengths ${ }^{20,21}$. Despite the attractions, the size effect is largely unexplored in lanthanide-doped upconversion nanoparticles, which represents an important family of optical materials characterized by large anti-Stokes shift, narrow emission bandwidths and long excited-state lifetimes.

Photon absorption and emission in upconversion nanoparticles are due to the lanthanide dopants localized on the lattice sites ${ }^{22,23}$. In principle, a high concentration of lanthanide dopants enhances upconversion processes as a result of an elevated capacity to sustain excitation energy ${ }^{24-27}$. However, a high lanthanide content also enhances energy migration through the crystal lattice, which usually leads to a depletion of the excitation energy ${ }^{24,28}$. To minimize nonradiative energy losses, energy migration is typically inhibited by doping low concentrations of lanthanide ions ${ }^{27,28}$ or by using special host lattices ${ }^{24}$. Currently, there lacks a general approach to maximize upconversion luminescence in stoichiometric lanthanide lattices.

In this work, we describe an investigation of energy migration in a nanosized $\mathrm{NaYbF}_{4}$ lattice. We demonstrate fine tuning of energy migration through controlling the dimensions of the crystal lattice. Our mechanistic investigation reveals a spatial confinement of energy migration that prevents energy loss to the crystal lattice and increases the local density of excitation energy. Through the use of $\mathrm{Tm}^{3+}$ ion as an energy accumulator, the excitation energy can be maximally amassed to generate intense ultraviolet emissions on near-infrared excitation. We show that the technological advancement may revolutionize the fabrication of cost effective and compact diode-pumped solid-state deep ultraviolet lasers that are useful for environmental, life science and industrial applications ${ }^{29}$.

\section{Results}

Synthesis and characterization. As a proof-of-concept experiment, we confined $\mathrm{Yb}^{3+}$ ions in the inner shell layer of a hexagonal phase $\mathrm{NaYF}_{4} @ \mathrm{NaYbF}_{4}: \mathrm{Tm} @ \mathrm{NaYF}_{4}$ host (Fig. 1a), which is known to render high upconversion efficiencies ${ }^{3}$. In our study, the concentration of $\mathrm{Tm}^{3+}$ was fixed at $1 \mathrm{~mol} \%$ to maximize upconversion emission in the ultraviolet region (Supplementary Fig. 1). We did not employ a $\mathrm{NaYbF}_{4}: \mathrm{Tm} @ \mathrm{NaYF}_{4}$ core-shell structure because existing synthetic protocols give essentially no access to sub- $10 \mathrm{~nm} \beta-\mathrm{NaYbF}_{4}$ nanoparticles of a tunable particle size, which is critical prerequisite for assessing the effect of confining energy migration on upconversion. Although $\mathrm{Yb}$-doped $\beta-\mathrm{NaYF}_{4}$ nanoparticles with small feature size can be synthesized by several complimentary methods ${ }^{23,30}$, pure $\beta-\mathrm{NaYbF}_{4}$ tends to form big particles (Supplementary Fig. 2a) due to rapid growth of the crystal ${ }^{31}$. Through the use of preformed $\mathrm{NaYF}_{4}$ core nanoparticle as a template, the growth of the $\mathrm{NaYbF}_{4}$ crystal can be effectively regulated, thereby offering exquisite control over the lattice dimensions (Supplementary Note 1). Note that it is also critical to enclose the $\mathrm{Yb}$ sublattice in an inert protection layer (that is, $\mathrm{NaYF}_{4}$ ) because a $\mathrm{NaYF}_{4} @ \mathrm{NaYbF}_{4}: \mathrm{Tm}$ core-shell structure yields luminescence that is substantially weak due to surface quenching (Supplementary Fig. 2b,c).

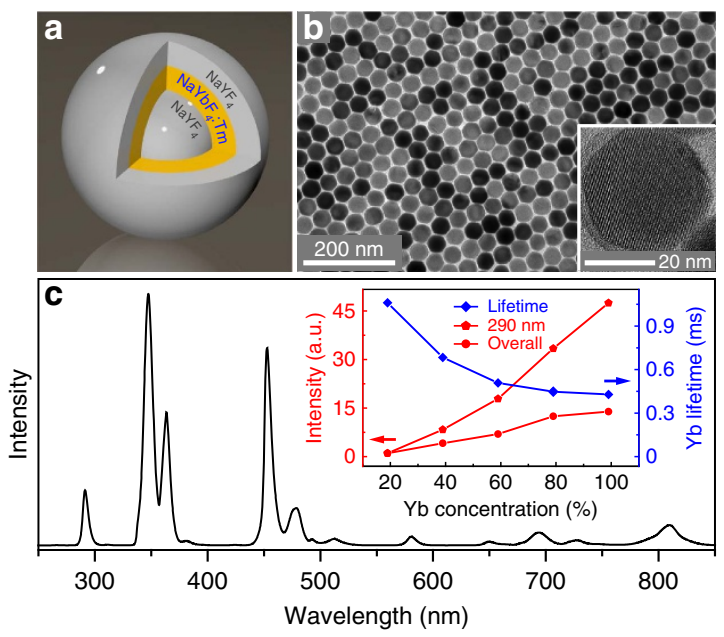

Figure 1 | Deep ultraviolet upconversion in core-shell-shell

nanoparticles. (a) Schematic design of a NaYF $4 @ \mathrm{NaYbF}_{4}: \mathrm{Tm}_{4} @ \mathrm{NaYF}_{4}$ core-shell-shell nanoparticle for confining the migration of excitation energy generated in the $\mathrm{Yb}^{3+}$ ions. (b) TEM image of the as-synthesized nanoparticles. Inset: high-resolution TEM image reveals single-crystalline nature of the particle. (c) Upconversion emission spectrum of the nanoparticles under $980 \mathrm{~nm}$ excitation ( $\mathrm{CW}$ laser diode, $20 \mathrm{~W} \mathrm{~cm}^{-2}$ ). Inset: ${ }^{2} \mathrm{~F}_{5 / 2}$ lifetime of $\mathrm{Yb}^{3+}$, emission intensity at $290 \mathrm{~nm}$ and integrated emission intensity over $250-850 \mathrm{~nm}$ range versus dopant concentration of $\mathrm{Yb}^{3+}$, respectively. Note that the solid lines are intended to guide the eye.

The nanoparticles were fabricated by a layer-by-layer epitaxial growth process (Supplementary Note 1). Transmission electron microscope (TEM) images (Fig. 1b) reveal a highly uniform morphology of the nanoparticles with an average size of $38 \mathrm{~nm}$. High-resolution TEM (inset of Fig. 1b) and X-ray powder diffraction (Supplementary Fig. 3) experiments confirm the single-crystalline nature of the as-synthesized nanoparticles with a hexagonal phase. To verify the formation of the multilayer structure, we intentionally doped $\mathrm{Gd}^{3+}$ ions in the inner shell layer to create a contrast under electron energy loss spectroscopy analysis. The difference in the elemental distribution of Y and Gd clearly indicates the presence of multiple core-shell interfaces (Supplementary Fig. 4).

Figure 1c shows a representative upconversion emission spectrum of $\mathrm{NaYF}_{4} @ \mathrm{NaYbF}_{4}: \mathrm{Tm} @ \mathrm{NaYF}_{4}$ nanoparticles on $980 \mathrm{~nm}$ excitation with a continuous wave (CW) laser diode at a power density of $20 \mathrm{~W} \mathrm{~cm}^{-2}$. The spectrum consists of characteristic emission peaks that can be assigned to ${ }^{1} \mathrm{I}_{6} \rightarrow{ }^{3} \mathrm{H}_{6}$ and ${ }^{3} \mathrm{~F}_{4}(290$ and $350 \mathrm{~nm}),{ }^{1} \mathrm{D}_{2} \rightarrow{ }^{3} \mathrm{H}_{6}$ and ${ }^{3} \mathrm{~F}_{4}$ (360 and $\left.450 \mathrm{~nm}\right)$, ${ }^{1} \mathrm{G}_{4} \rightarrow{ }^{3} \mathrm{H}_{6}$ and ${ }^{3} \mathrm{~F}_{4}$ (475 and $650 \mathrm{~nm}$ ) and ${ }^{3} \mathrm{H}_{4} \rightarrow{ }^{3} \mathrm{H}_{6}(800 \mathrm{~nm})$ transitions of $\mathrm{Tm}^{3+}$, respectively. Both the violet and overall emissions surpass that of the $\mathrm{NaYF}_{4} @ \mathrm{NaYbF}_{4}: \mathrm{Tm} / \mathrm{Y} @ \mathrm{NaYF}_{4}$ counterparts comprising lower $\mathrm{Yb}^{3+}$ contents (inset of Fig. 1c and Supplementary Figs 5 and 6). Notably, $\mathrm{Tm}^{3+}$ emission at $290 \mathrm{~nm}$ originating from a five-photon upconversion declined by over 45 -fold when the $\mathrm{Yb}^{3+}$ concentration dropped to $19 \mathrm{~mol} \%$, which in conventional systems typically produces the maximum emission of $\mathrm{Tm}^{3+}$ ions ${ }^{44,28}$.

Confinement of energy migration. We attribute the observations to confined migration of excitation energy within the nanoshells, which prevents the excitation energy from travelling a long distance at a high $\mathrm{Yb}^{3+}$ concentration $(99 \mathrm{~mol} \%)$. The absence of long-distance energy migration is likely to suppress energy loss to the crystal lattice accounting for luminescence quenching. Furthermore, the localization of excitation energy raises the rate 
of energy transfer to a nearby $\mathrm{Tm}^{3+}$ activator, which facilitates the multiphoton upconversion process.

An assessment of a series of $\mathrm{NaYF}_{4} @ \mathrm{NaYbF}_{4}: \mathrm{Tm}_{\mathrm{N}} @ \mathrm{NaYF}_{4}$ nanoparticles of varying inner shell thickness from 1 to $17 \mathrm{~nm}$ verified the spatial confinement of energy migration (Supplementary Fig. 7). Luminescence decay studies reveal a markedly lengthened lifetime of the $\mathrm{Yb}^{3+}$ by a factor of over nine with decreasing inner shell thickness from 17 to $1 \mathrm{~nm}$ (Supplementary Fig. 8a), confirming the suppression of energy loss to the host lattice in thin shells. In contrast, the decay time of localized $\mathrm{Tm}^{3+}$ transition was only increased by less than twofold for the same series of samples (Supplementary Fig. 8b), suggesting that the defect density in the host lattice were marginally modified. Therefore, the suppressed depletion of excitation energy of $\mathrm{Yb}^{3}+$ may be dominantly ascribed to the spatial confinement of energy migration, which reduces the quantity of defects accessible to the Yb sublattice. In line with the reduced energy loss, we observed a steady enhancement of upconversion emission, especially the part in the $280-356 \mathrm{~nm}$ range that originates from the five-photon process, accompanied by a decrease of the inner shell thickness (Fig. 2a; Supplementary Fig. 9). The slight drop in the ratio of five-photon upconversion to overall emission for substantially thin shells (that is, 1 and $2 \mathrm{~nm}$ ) can be attributed to the reduced amount of $\mathrm{Yb}^{3+}$ ions in the vicinity of a $\mathrm{Tm}^{3+}$ activator (Fig. 2b), which limits the quantity of energy that can be captured by a $\mathrm{Tm}^{3+}$ activator in a photo cycle. It is noted that the spatial confinement of energy migration also plays a role in cubic phase $\mathrm{NaYbF}_{4}: \mathrm{Tm}$ (1\%)@NaYF 4 core-shell nanoparticles (Supplementary Fig. 10) ${ }^{32}$, implying that the geometry of the host lattice is not a formative factor in restraining the migration of excitation energy.

To shed more light on energy migration in the core-shell-shell nanostructure, we calculated the probability distribution function of excitation energy as a function of space within the $\mathrm{Yb}$ shell. For simplification, we assumed that the excitation energy randomly hops in the inner shell layer through the Dexter energy transfer (Supplementary Note 2). As shown in Fig. 2c, the energy migrates to smaller areas with decreasing thickness of the $\mathrm{Yb}$ shell from 12 to 6 and $3 \mathrm{~nm}$, supporting reduced coupling of excitation energy to defects. The high probability of finding the excitation energy in a thin $\mathrm{Yb}$ shell further validates a favourable energy transfer to an adjacent $\mathrm{Tm}^{3+}$ activator.

Lasing through upconversion. To facilitate the use of the upconversion nanoparticles as gain media for lasing applications, we further developed a $\mathrm{Gd}^{3}+$ doping method for optimizing the optical properties (Supplementary Fig. 11). We used a 3-nm inner shell for the study due to a high-intensity ratio of five-photon upconversion emission and a relatively low mass ratio of the optically inert $\mathrm{NaYF}_{4}$ layers. $\mathrm{Gd}^{3+}$ ions are able to extract the excitation energy of $\mathrm{Tm}^{3}+$ ions and generate a new emission peak centred at around $311 \mathrm{~nm}$, owing to the reasonably matched energy levels (that is, ${ }^{6} \mathrm{P}_{7 / 2}$ level of $\mathrm{Gd}^{3+}$ and ${ }^{1} \mathrm{I}_{6}$ level of $\mathrm{Tm}^{3+}$ ) (Fig. 3a,b). Importantly, the large energy gap $\left(32,200 \mathrm{~cm}^{-1}\right)$ in $\mathrm{Gd}^{3+}$ favours the preservation of the excitation energy as supported by time decay studies (Fig. 3c). The long-lived excited state contributes to high optical gains of around $150 \mathrm{~cm}^{-1}$ through five-photon upconversion (Fig. 3d), which is comparable to that of the GaN-based semiconductor quantum wells operating in deep ultraviolet at room temperature $^{33,34}$. The optical gain is also two orders of magnitude higher than that of a conventional $\mathrm{Yb} / \mathrm{Tm}$-based system operating at $650 \mathrm{~nm}$ through three-photon upconversion ${ }^{35}$. Moreover, efficient emission is attained at a high $\mathrm{Gd}^{3+}$ concentration (30 mol\%; Supplementary Fig. 12), which provides abundant
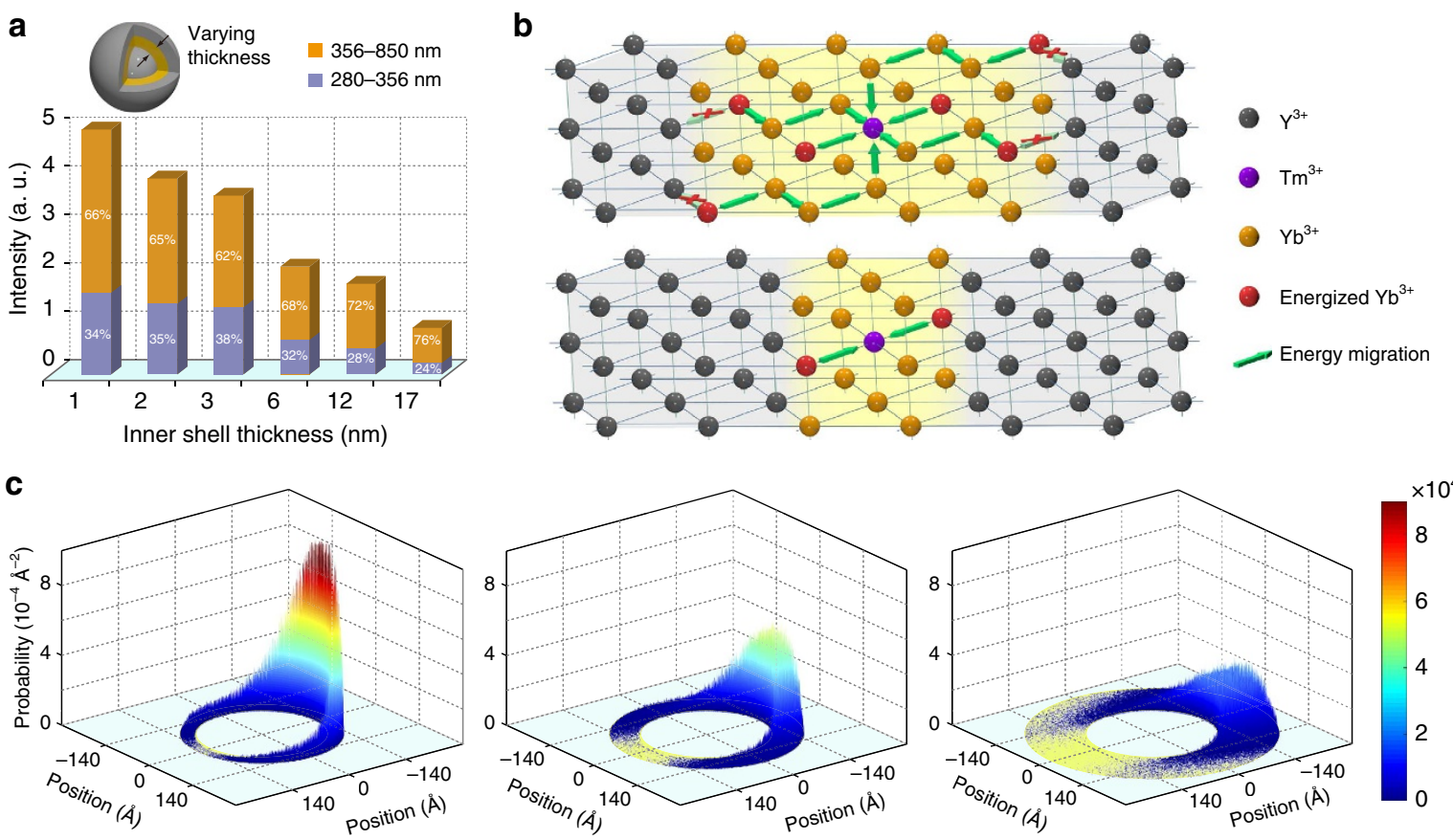

Figure 2 | The effect of inner shell thickness on upconversion. (a) Upconversion emission intensity versus inner shell thickness (1-17 nm). The emission intensities were calculated by integrating the spectral intensity of the emission spectra that are normalized to the absorption of $\mathrm{Yb}^{3+}$ at $980 \mathrm{~nm}$.

(b) Schematic illustration showing proposed energy transfer from $\mathrm{Yb}^{3+}$ to $\mathrm{Tm}^{3+}$ in $\mathrm{Yb}$-sublattice of varying dimensions. Note that only partial lattice sites are shown for clarification. (c) The probability of finding the excitation energy on the equatorial section of core-shell-shell nanoparticles of varying inner shell thickness. With increasing inner shell thickness (from left to right panels), the energy migrates to a larger area and the probability of finding the excitation energy in the vicinity of the starting point drops significantly. 

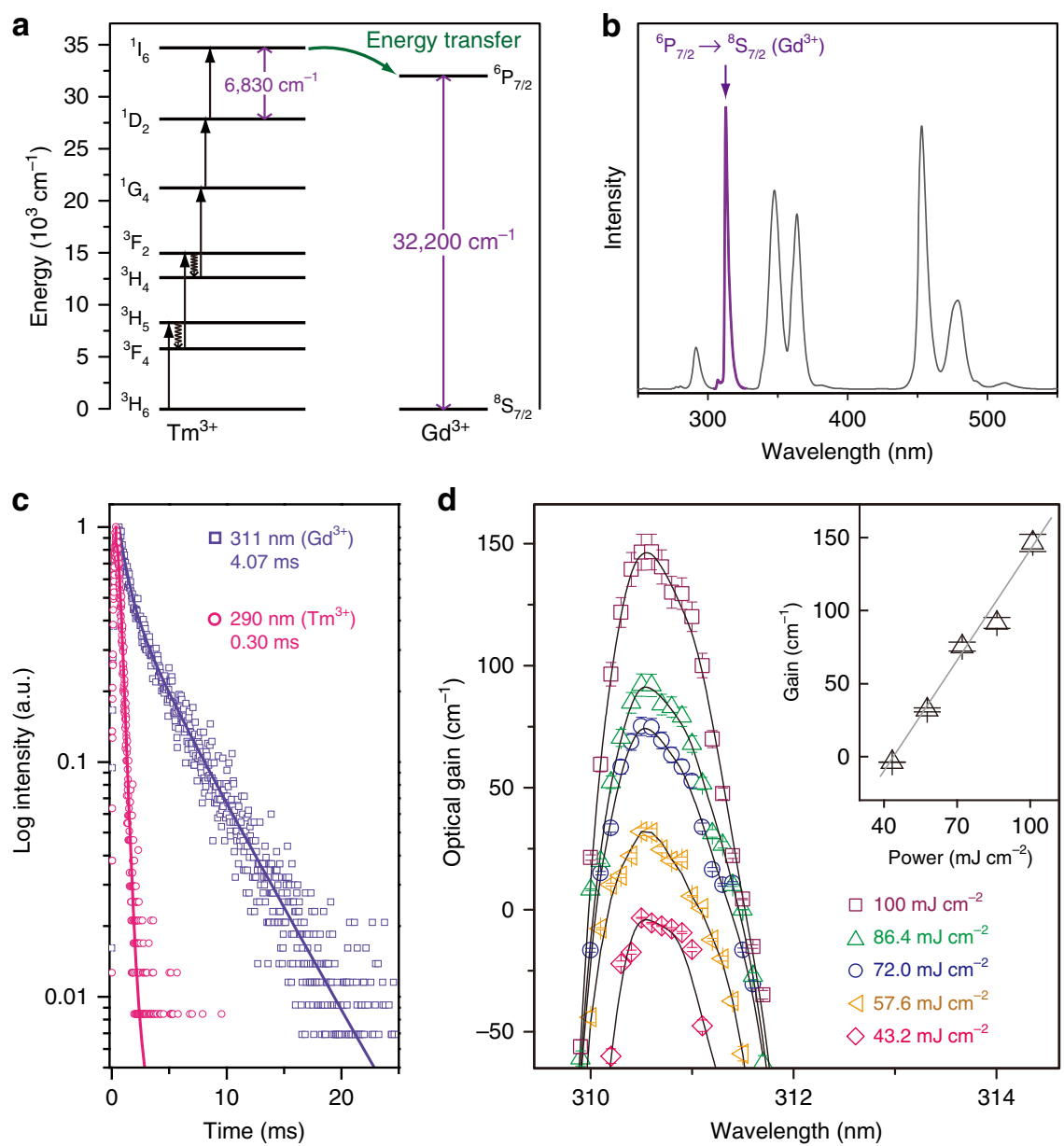

Figure 3 | The effect of $\mathbf{G d}^{\mathbf{3}+}$ doping on the deep ultraviolet upconversion. (a) Simplified energy level diagram showing the energy gaps in $\mathrm{Tm}^{3+}$ and $\mathrm{Gd}^{3+}$ activators, respectively. (b) Upconversion emission spectra of the core-shell-shell nanoparticles co-doped $\mathrm{Gd}^{3+}\left(30 \mathrm{~mol}^{2}\right)$ in the inner shell layer ( $\mathrm{CW}$ laser diode, $20 \mathrm{~W} \mathrm{~cm}^{-2}$ ). (c) A comparison of the excited state lifetime between ${ }^{1} I_{6}$ state of $\mathrm{Tm}^{3+}$ and ${ }^{6} \mathrm{P}_{7 / 2}$ state of $\mathrm{Gd}^{3+}$ in the $\mathrm{NaYF}_{4} @ \mathrm{NaYbF}_{4}: \mathrm{Tm} / \mathrm{Gd}(1 / 30 \%) @ \mathrm{NaYF}_{4}$ core-shell-shell nanoparticles. (d) Gain spectra of the nanoparticles in $\mathbf{b}$ as a function of excitation power (pulse laser). The inset gives the corresponding optical gain versus pump power at a wavelength of $310.5 \mathrm{~nm}$. The straight line is the linear regression of the measured data. Error bars shown represent the s.d.'s from five sets of repeated measurements.

carriers to sustain optical gains at high excitation powers without saturation (Fig. 3d).

To realize lasing emission, we constructed a five-pulse pumping scheme to excite the upconversion process (Fig. 4a). The pulse excitation scheme is primarily intended to alleviate the problems of catastrophic optical damage and the thermal effects associated with CW excitation, which terminates upconversion lasing actions. Furthermore, the five-pulse system is advantageous over the single-pulse system for pumping the multiphoton upconversion (Supplementary Fig. 13), as a result of improved alignment with the excitation process where the absorption of photons occurs sequentially ${ }^{36}$.

The laser cavity was fabricated by coating a drop of silica resin containing the nanoparticles onto a standard optical fibre. Driven by surface tension, the silica resin tends to form a bottle-like microresonator, which supports whispering gallery modes at a thin equatorial ring near the surface of the microresonator (Fig. 4b). Notably, the emission features such as mode spacing and threshold pump power of the microresonator can be readily tuned by controlling the diameter $\left(D_{\mathrm{m}}\right)$ of the resonator (Fig. 4b) ${ }^{37,38}$, which provides a general platform for assessing the optical characteristics of the upconversion nanoparticles.

We validated the lasing action by measuring the optical emission in a typical microresonator $\left(D_{\mathrm{m}}=75 \mu \mathrm{m}\right)$ under excitation of varying powers at room temperature. The light input-output curve shown in Fig. 4c exhibits a well-defined nonlinear excitation power-dependent behaviour with three distinct regions separated by two threshold pump powers (that is, $P_{\mathrm{a}}=\sim 78 \mathrm{~mJ} \mathrm{~cm}^{-2}$ and $\left.P_{\mathrm{th}}=\sim 86 \mathrm{~mJ} \mathrm{~cm}^{-2}\right)$. This $S$-like spectrum clearly indicates a transition from a spontaneous emission to an amplified spontaneous emission and to a lasing emission. Figure $4 \mathrm{~d}$ shows the corresponding emission spectra under various pumping powers. At low pump power $\left(<P_{\mathrm{a}}\right)$, a relatively broad spontaneous band is observed. As the excitation power increases slightly above $P_{\mathrm{a}}$, a sharp peak ascends from the emission spectrum. Through further increases in the excitation power above $P_{\text {th }}$, well-defined sharp peaks with a linewidth $<0.11 \mathrm{~nm}$ emerge from the spectrum. The measured mode spacing $(0.25-0.27 \mathrm{~nm})$ is in good agreement with the theoretical value $(\sim 0.26 \mathrm{~nm})$, confirming that lasing emissions have been achieved. Notably, single-mode lasing was also obtained by using a thinner microresonator $\left(D_{\mathrm{m}}=20 \mu \mathrm{m}\right.$; Fig. 4e). The single-mode emission is a result of a relatively large mode spacing $(\sim 1.0 \mathrm{~nm})$ with respect to the full width at half maximum of the resonant frequency. This narrow gain bandwidth is a unique signature of the upconversion nanoparticles and unlikely to be realized from semiconductor nanostructures. The $Q$ factor, which is defined as the ratio of 


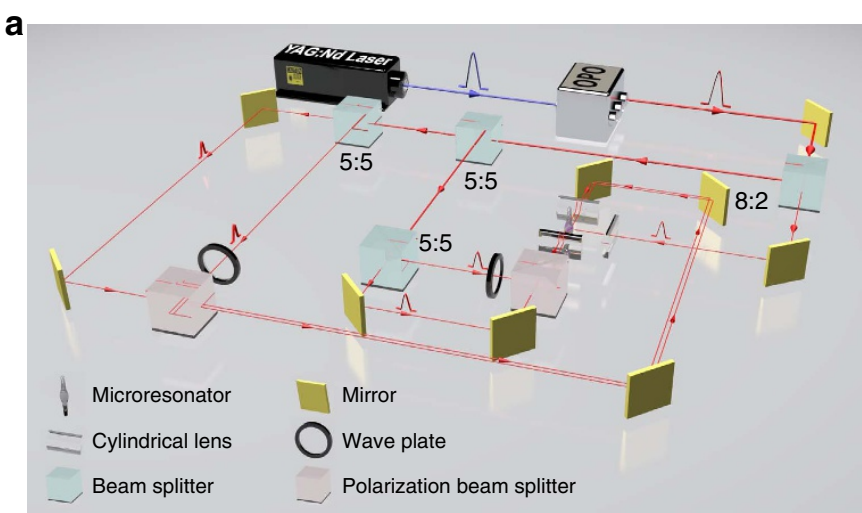

C

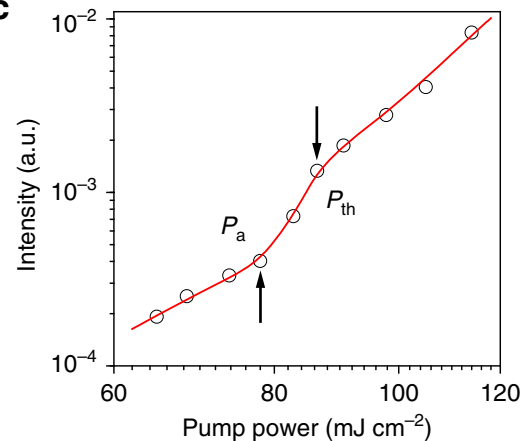

d

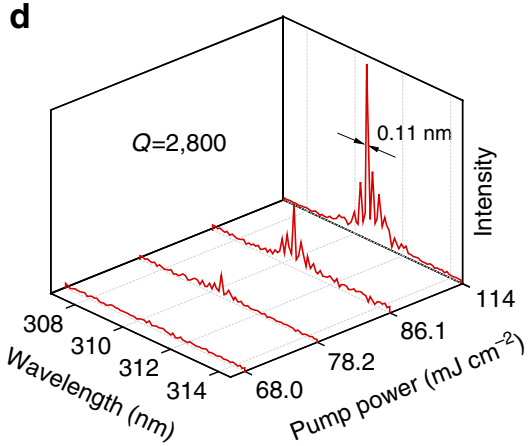

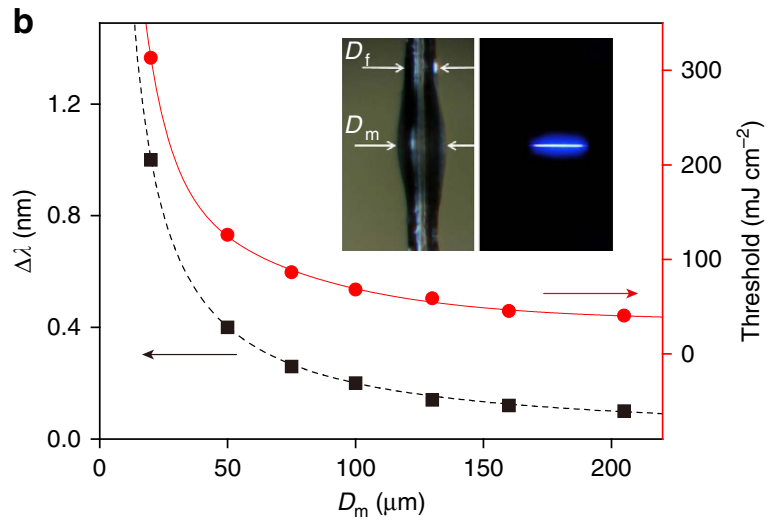

e

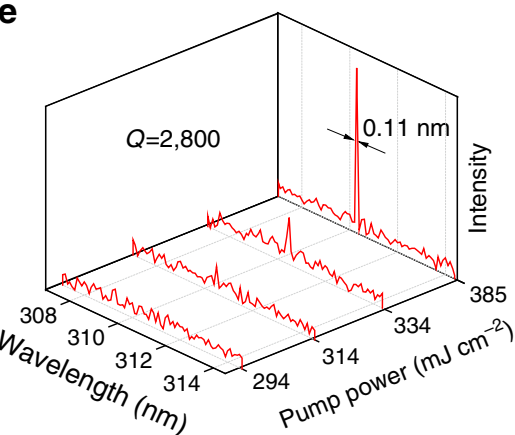

Figure 4 | Upconversion lasing characteristics of the microresonator. (a) Schematic diagram of the optical set-up for the five-pulse excitation scheme. (b) Plots of measured $\Delta \lambda$ and $P_{\mathrm{th}}$ of the microresonator as a function of $D_{\mathrm{m}}$. The red and black lines are fitted and calculated curves, respectively. The insets show photographs of the microresonator with and without optical excitation. $D_{f}$ and $D_{m}$ denote the diameters of the fibre and microcavity, respectively. (c) Logarithmic plot of output intensity versus excitation power of a microresonator with $D_{\mathrm{m}}=75 \mu \mathrm{m}$. The red line is fitted curve. (d) The corresponding lasing spectra at different excitation power $\left(D_{\mathrm{m}}=75 \mu \mathrm{m}\right)$. (e) Single mode lasing spectra measured from a microresonator with $D_{\mathrm{m}}=20 \mu \mathrm{m}$.

the resonant frequency to its full width at half maximum, was estimated to be $\sim 2,800$, revealing the high quality of the upconversion-based laser system. It is also worth noting that the lasing emission can be readily extended to violet and blue spectral regions with the same upconversion nanoparticles (Supplementary Fig. 14).

\section{Discussion}

Our investigation of energy migration in nanostructured hosts highlights an innovative strategy to manipulating optical transitions in lanthanide-doped upconversion nanoparticles. In addition, it initiates a novel tactic to obtain effective upconversion laser materials in deep ultraviolet regime with very narrow optical gain bandwidth to support single-mode excitation. Emission, in principle, can be tuned to shorter wavelengths (that is, well below $300 \mathrm{~nm}$ ) by further refining the upconversion process. Hence, our study will lead to the development of near-infrared diode-pumped deep ultraviolet lasers which can avoid the difficulty of shifting the operating wavelength of GaN-based laser diodes below $300 \mathrm{~nm}$ (ref. 39), evade using nonlinear optical crystal that requires tight control in optical alignment, antireflective coating and environmental control and adopt inexpensive $Q$-switched near-infrared diode as the pumping source to construct compact, deep ultraviolet lasers for unexplored applications in the fields of information technology, biomedicine and biophotonics.

\section{Methods}

Nanoparticle synthesis. We synthesized the core-shell-shell nanoparticles using the method described in ref.16. Additional experimental details are provided in the Supplementary Note 1.
Optical gain measurement. Net optical gain of the nanoparticle colloid was measured using variable stripe length method ${ }^{40}$. The longer side of a quartz cuvette filled with nanoparticle colloid was excited by a pump stripe with width and length of $\sim 30 \mu \mathrm{m}$ and $L$, respectively in the orientation perpendicular to the length of the cuvette. Photoluminescence intensity emitted from the shorter side of the cuvette, $I_{\text {tot }}(\lambda)$, was recorded by the monochromator set-up. The net optical gain, $G(\lambda)$, was deduced by fitting $I_{\text {tot }}(L, \lambda)=I_{\mathrm{sp}}(\lambda)[\exp (G(\lambda) L)-1] / G(\lambda)$ with the measured values of $I_{\text {tot }}(\lambda)$, where $\lambda$ is the wavelength and $I_{\text {sp }}(\lambda)$ is the spontaneous emission intensity.

Five-pulse excitation scheme. The optical set-up consists of a Powerlite DLS 9010 Q-switched Nd:YAG laser and a continuum Panther EX optical parametric oscillator. A $980-\mathrm{nm}$ laser pulse $(6 \mathrm{~ns}, 10 \mathrm{~Hz})$ with a diameter of $\sim 10 \mathrm{~mm}$ was generated from the optical parametric oscillator system under the excitation of the $\mathrm{Nd}$ :YAG laser. By splitting the $980-\mathrm{nm}$ pulse into five equal-power pulses through the use of four beam splitters (that is, one $80 / 20$ and three $50 / 50$ beam splitters), we can obtain a five-pulse (time delay between adjacent pulses is $10 \mathrm{~ns}$ ) laser beam. This is possible because the pulses are forced to travel in five different distances to obtain a time delay of $10 \mathrm{~ns}$ between the adjacent pulses. These five pulses are then combined through two polarization-dependent beam splitters and two $\lambda / 2$ waveplates to form three laser beams. All the laser beams, which are spatially overlapped, are focussed onto a sample through three cylindrical lenses to form a pump stripe of width equal to $\sim 30 \mu \mathrm{m}$. Photoluminescence emission from the sample was collected and analysed by an optical fibre (core diameter of $400 \mu \mathrm{m}$ ) coupled to an Oriel MS257 monochromator attached with a photomultiplier tube. The spectral resolution of the monchromator is about $0.1 \mathrm{~nm}$.

Fabrication and excitation of microresonators. For the fabrication of the bottle-like microresonator, a bared standard optical fibre was coated with a tiny drop of nanoparticles and silica resin mixture. The prolate surface-tention-induced microresonator was then solidified in an arid and clean atmosphere. The surrounding temperature of the sample was kept at $23^{\circ} \mathrm{C}$ to avoid deformation due to the influence of thermal effects. Whispering gallery modes can be excited by optically pumping the equatorial zone of the microresonator. Notably, the pump stripe is oriented perpendicular to the length of the fibre. Light emitted from the surface of the microresonator can be collected through an optical fibre. 


\section{References}

1. Yu, J. H. et al. High-resolution three-photon biomedical imaging using doped ZnS nanocrystals. Nat. Mater. 12, 359-366 (2013).

2. Astruc, M.-C. \& Astruc, D. Gold nanoparticles: assembly, supramolecular chemistry, quantum-size-related properties, and applications toward biology, catalysis, and nanotechnology. Chem. Rev. 104, 293-346 (2004).

3. Haase, M. \& Schafer, H. Upconverting nanoparticles. Angew. Chem. Int. Ed. 50, 5808-5829 (2011).

4. Hola, K. et al. Carbon dots-emerging light emitters for bioimaging, cancer therapy and optoelectronics. Nano Today 9, 590-603 (2014).

5. Chen, X., Liu, L., Yu, P. Y. \& Mao, S. S. Increasing solar absorption for photocatalysis with black hydrogenated titanium dioxide nanocrystals. Science 331, 746-750 (2011).

6. Carter, K. P., Young, A. M. \& Palmer, A. E. Fluorescent sensors for measuring metal ions in living systems. Chem. Rev. 114, 4564-4601 (2014)

7. Idris, N. M., Jayakumar, M. K., Bansal, A. \& Zhang, Y. Upconversion nanoparticles as versatile light nanotransducers for photoactivation applications. Chem. Soc. Rev. 44, 1449-1478 (2015).

8. Su, L. T. et al. Photon upconversion in hetero-nanostructured photoanodes for enhanced near-infrared light harvesting. Adv. Mater. 25, 1603-1607 (2013).

9. Yang, D. et al. Current advances in lanthanide ion $\left(\mathrm{Ln}^{3+}\right)$-based upconversion nanomaterials for drug delivery. Chem. Soc. Rev. 44, 1416-1448 (2015).

10. Liu, J. et al. Metal-free efficient photocatalyst for stable visible water splitting via a two-electron pathway. Science 347, 970-974 (2015).

11. Sang, Y. et al. From UV to near-infrared, WS 2 nanosheet: a novel photocatalyst for full solar light spectrum photodegradation. Adv. Mater. 27, 363-369 (2015).

12. Alivisatos, A. Semiconductor clusters, nanocrystals, and quantum dots. Science 271, 933-937 (1996).

13. Novoselov, K. S. et al. Novoselov-electric field effect in atomically thin carbon films. Science 306, 666-669 (2004).

14. Deng, R. et al. Temporal full-colour tuning through non-steady-state upconversion. Nat. Nanotechnol 10, 237-242 (2015).

15. Sun, Y., Zhu, X., Peng, J. \& Li, F. Core-shell lanthanide upconversion nanophosphors as four-modal probes for tumor angiogenesis imaging. ACS Nano 7, 11290-11300 (2013).

16. Wen, H. et al. Upconverting near-infrared light through energy management in core-shell-shell nanoparticles. Angew. Chem. Int. Ed. 52, 13419-13423 (2013).

17. Sun, L. D., Wang, Y. F. \& Yan, C. H. Paradigms and challenges for bioapplication of rare earth upconversion luminescent nanoparticles: small size and tunable emission/excitation spectra. Acc. Chem. Res. 47, 1001-1009 (2014).

18. Li, X., Zhang, F. \& Zhao, D. Lab on upconversion nanoparticles: optical properties and applications engineering via designed nanostructure. Chem. Soc. Rev. 44, 1346-1378 (2015).

19. Tu, L., Liu, X., Wu, F. \& Zhang, H. Excitation energy migration dynamics in upconversion nanomaterials. Chem. Soc. Rev. 44, 1331-1345 (2015).

20. Rossetti, R., Nakahara, S. \& Brus, L. E. Quantum size effects in the redox potentials, resonance Raman spectra, and electronic spectra of CdS crystallites in aqueous solution. J. Chem. Phys. 79, 1086 (1983).

21. Bruchez, M., Moronne, M., Gin, P., Weiss, S. \& Alivisatos, A. P. Semiconductor nanocrystals as fluorescent biological labels. Science 281, 2013-2016 (1998).

22. Wu, S. et al. Non-blinking and photostable upconverted luminescence from single lanthanide-doped nanocrystals. Proc. Natl Acad. Sci. USA 106, 10917-10921 (2009)

23. Gargas, D. J. et al. Engineering bright sub-10-nm upconverting nanocrystals for single-molecule imaging. Nat. Nanotechnol 9, 300-305 (2014).

24 . Wang, J. et al. Enhancing multiphoton upconversion through energy clustering at sublattice level. Nat. Mater. 13, 157-162 (2013).

25. Punjabi, A. et al. Amplifying the red-emission of upconverting nanoparticles for biocompatible clinically used prodrug-induced photodynamic therapy. ACS Nano 8, 10621-10630 (2014).

26. Shen, J. et al. Tunable near infrared to ultraviolet upconversion luminescence enhancement in $\left(\alpha-\mathrm{NaYF}_{4}: \mathrm{Yb}, \mathrm{Tm}\right) / \mathrm{CaF}_{2}$ core/shell nanoparticles for in situ real-time recorded biocompatible photoactivation. Small 9, 3213-3217 (2013).

27. Mahalingam, V., Vetrone, F., Naccache, R., Speghini, A. \& Capobianco, J. A. Colloidal $\mathrm{Tm}^{3+} / \mathrm{Yb}^{3+}$-doped $\mathrm{LiYF}_{4}$ nanocrystals: multiple luminescence spanning the UV to NIR regions via low-energy excitation. Adv. Mater. 21, 4025-4028 (2009).
28. Krämer, K. W. et al. Hexagonal sodium yttrium fluoride based green and blue emitting upconversion phosphors. Chem. Mater. 16, 1244-1251 (2004).

29. Boardman, E. A. et al. Deep ultraviolet (UVC) laser for sterilisation and fluorescence applications. Sharp Tech. Rep. 104, 31-35 (2012).

30. Ostrowski, A. D. et al. Controlled synthesis and single-Particle imaging of bright, sub-10 nm lanthanide-doped upconverting nanocrystals. ACS Nano 6, 2686-2692 (2012).

31. Wang, F. et al. Simultaneous phase and size control of upconversion nanocrystals through lanthanide doping. Nature 463, 1061-1065 (2010)

32. Chen, G., Ohulchanskyy, T. Y., Kumar, R., Ågren, H. \& Prasad, P. N. Ultrasmall monodisperse $\mathrm{NaYF}_{4}: \mathrm{Yb}^{3+} / \mathrm{Tm}^{3+}$ nanocrystals with enhanced near-infrared to near-infrared upconversion photoluminescence. ACS Nano 4, 3163-3168 (2010).

33. Witzigmann, B. L. et al. Analysis of temperature-dependent optical gain in GaN-InGaN quantum-well structures. IEEE Photon. Technol. Lett 18, 1600-1602 (2006).

34. Pecora, E. F., Sun, H., Negro, L. D. \& Moustakas, T. D. Deep-UV optical gain in AlGaN-based graded-index separate confinement heterostructure. Opt. Mater. Express 5, 809-817 (2015).

35. Haro-González, P., Martín, I. R., Lahoz, F. \& Capuj, N. E. Optical gain by upconversion in $\mathrm{Tm}-\mathrm{Yb}$ oxyfluoride glass ceramic. Appl. Phys. B 104, 237-240 (2010).

36. Zhu, H. et al. Amplified spontaneous emission and lasing from lanthanidedoped up-conversion nanocrystals. ACS Nano 7, 11420-11426 (2013).

37. Vahala, K. J. Optical microcavities. Nature 424, 839-846 (2003).

38. Pöllinger, M., O'Shea, D., Warken, F. \& Rauschenbeutel, A. Ultrahigh-Q tunable whispering-gallery-mode microresonator. Phys. Rev. Lett. 103, 053901 (2009).

39. Yoshida, H., Yamashita, Y., Kuwabara, M. \& Kan, H. A 342-nm ultraviolet AlGaN multiple-quantum-well laser diode. Nat. Photon 2, 551-554 (2008).

40. Valenta, J., Pelant, I. \& Linnros, J. Waveguiding effects in the measurement of optical gain in a layer of Si nanocrystals. Appl. Phys. Lett. 81, 1396 (2002).

\section{Acknowledgements}

This work was supported by the Research Grants Council of Hong Kong (CityU 109413, 21300014 and PolyU 153036/14P) and the National Natural Science Foundation of China (Nos. 21303149, 51332008 and 21403182).

\section{Author contributions}

X.C., L.J., S.F.Y. and F.W. conceived the project and wrote the paper. X.C., W.K., T.S. and F.W synthesized and characterized the nanoparticles. L.J., W.Z. and S.F.Y. fabricated and tested the lasers. X.L. and J.F. solved the probability of finding the excitation energy. All authors contributed to the analysis of this manuscript.

\section{Additional information}

Supplementary Information accompanies this paper at http://www.nature.com/ naturecommunications

Competing financial interests: The authors declare no competing financial interests

Reprints and permission information is available online at http://npg.nature.com/ reprintsandpermissions/

How to cite this article: Chen, X. et al. Confining energy migration in upconversion nanoparticles towards deep ultraviolet lasing. Nat. Commun. 7:10304 doi: 10.1038/ncomms10304 (2016).

This work is licensed under a Creative Commons Attribution 4.0 International License. The images or other third party material in this article are included in the article's Creative Commons license, unless indicated otherwise in the credit line; if the material is not included under the Creative Commons license, users will need to obtain permission from the license holder to reproduce the material. To view a copy of this license, visit http://creativecommons.org/licenses/by/4.0/ 